\title{
CARCINOID SYNDROME-CASE REPORT
}

*Mrs. Kripa Angeline. A \& **Mrs. Janifer Xavier Albertina. J

\section{INTRODUCTION:}

Carcinoid syndrome is a set of symptoms caused by some carcinoid tumors, which grow out of cells of the Endocrine System. These tumors produce too many hormones (e.g., Serotonin) which cause the symptoms of Carcinoid Syndrome.

\section{Carcinoid tumor was first} characterized by Siegfried Oberndorfer, a German pathologist who coined the term "Karzinoide" or "carcinoma-like", to describe the unique feature of behaving like a benign tumor though having a microscopic malignant appearance. Carcinoid tumor is a slow-growing type of Neuroendocrine tumor originating in the cells of the neuroendocrine system most commonly presenting in the midgut at the level of the ileum or in the appendix. Carcinoid tumors are APUDomas (Amine Precursor Uptake and Decarboxylation)that arise from the enterochromaffin cells throughout the gut. Over $2 / 3$ rds of the tumors are found in the Gastrointestinal tract.

\section{Incidence:}

- $10 \%$ of people who have carcinoid tumors develop Carcinoid Syndrome.

- Carcinoid syndrome occurs in $8 \%$ of 8876 patients with carcinoid tumor.

- Men are more prone than women (46 to 59\% a presentation and 46-61\% during course of the disease) Depending on location of carcinoid tumour the common sites are asfollows:

\begin{tabular}{|c|c|c|}
\hline $\begin{array}{l}\text { Tumor } \\
\text { location }\end{array}$ & $\begin{array}{l}\text { At } \\
\text { Presentation }\end{array}$ & $\begin{array}{l}\text { During the } \\
\text { course of } \\
\text { disease }\end{array}$ \\
\hline Foregut & $5-9 \%$ & $2-33 \%$ \\
\hline Midgut & $78-87 \%$ & $60-87 \%$ \\
\hline Hindgut & $1-5 \%$ & $1-8 \%$ \\
\hline Unknown & $2-11 \%$ & $2-15 \%$ \\
\hline
\end{tabular}

\section{Etiology :}

Caused by a carcinoid tumor which secretes serotonin or other chemicals into the blood stream. But the cause of carcinoid tumor is unknown.Most tumors leading to carcinoid syndrome originate in the intestine and the syndrome usually does not appear until there is metastasis to the liver.

\section{Aggravating Factors:}

Stress, Physical exertion, Alcohol consumption, Food items like cheese, meat and tyramine containing foods .

\section{Pathophysiology}

Carcinoid tumor in any part of the bodySerotonin, a vasoactive substance is secreted by the carcinoid tumor, which causes flushing results from secretion of Kallikrein, the enzyme that catalyses the conversion of kininogen to lysyl-bradykinin. Lysyl-bradykinin is converted to bradykinin, the most powerful vasodilator. The chemicals

\footnotetext{
*Assoc. Prof. \& **Asst. Lect. Kasturba Gandhi Nursing College, SBV, Puducherry.
} 
enter the systemic circulation. The chemicals enter the liver where it is neutralized without manifestations.If tumors arise in the lung, bronchi, liver and metastasizes to the liver.Have direct access to the systemic circulation before being metabolized.Exhibition of symptoms of carcinoid syndrome.Carcinoid syndrome manifestations occur.

\section{Signs and Symptoms:}

$>$ Diarrhea

Flushing of the skin or face (dry and not associated with sweating)

$>$ Heart palpitations

$>$ Stomach cramps

$>$ Shortness of breath

$>$ Wheezing

$>$ Pain

$>$ Pellagra

\section{Diagnosis:}

As the symptoms coincide with common conditions like Irritable Bowel Syndrome, the diagnosis is misinterpretated. The common tests are :

- Serum Analysis: Serum analysis of chromogranin A, a glycoprotein that is secreted with other hormones by neuroendocrine tumors, appears to be the most promising, with specificity approaching 95 percent and sensitivity for carcinoid tumors approaching 80 percent.

- Urine analysis: Urine 5-HIAA measures the amount of the hormone serotonin being made in the body over a period of $24 \mathrm{hrs}$.

- Imaging studies :

$>$ Octreoscan,X-Ray, CT scan and MRI Scan.

$>$ Endoscopy . and ultrasonogram
Biopsy may reveal hepatic lesions

\section{Treatment:}

* Removing or shrinking the carcinoid tumor. In general treatments fall into one of the following categories:

* Surgery is the usually the first choice of treatment. The type of surgery used depends on the location of the tumor and its size and the state of metastasis.

* Medications are used for symptomatic relief of carcinoid syndrome when the tumor cannot be resected by surgery. The most common drug of choice is octreotide (sandostatin) which relieves flushing and diarrhea and also helps to slow the growth of tumors.

* Alpha - Interferon

* Chemotherapy

* Radiation therapy.

\section{Prognosis:}

As the tumors are slow growing it is possible to treat tumors at this stage. It is usual for people to live for 10 to 15 years with carcinoid syndrome.But the most common cause of carcinoid syndrome is metastatic liver disease arising from a small bowel carcinoid tumor. For these patients, the prognosis is uniformly poor.

\section{Complications :}

Carcinoid heart disease, Bowel obstruction, Carcinoid crisis.

\section{Nursing Management :}

Nursing management of carcinoid syndrome is discussed along with case report. 


\section{CASE REPORT:}

Mr. A, 45yrs/Male, got admitted inMGMC\&RI on 25/06/2014 with the complaints of Giddiness, drowsiness with slurred incoherent speech, non projectile not blood/bile stained vomiting containing food particles associated with abdominal pain around umbilicus, 3 episodes of non blood stained loose stools since 2 days. Patient history revealed recurrent hospitalization for the complaints of giddiness, vomiting, loose stools and decreased appetite, over the last 10 months which was relieved on hospitalization. He is a known case of Type II Diabetes Mellitus for the past 6 months and is on medication and he had present complaints of decreased appetite, gradual lowering of weight, generalized weakness, breathlessness and diffuse headache. On examination there were no any significant findings.Blood investigations showed elevated blood glucose values of $220 \mathrm{mg} / \mathrm{dl}$ and decreased serum potassium values of 2.6 $\mathrm{mEq} / \mathrm{dl}$.Chest $\mathrm{x}$-ray study showed no abnormalities.Ultrasonography of abdomen revealed the evidence of tumor of the liver. Liver biopsy revealed the evidence of Carcinoid tumor with hepatic lesions treated with Tab. Rantac $150 \mathrm{mg}$ bd ,Tab. Vertin 16 mg bd, NS@75 ml/hr.

\section{NURSING CARE :}

Nursing management of Mr. A with Carcinoid syndrome is presented as nursing process approach based on the problems and needs identified.

\section{Nursing assessment :}

Complaints of breathlessness, decreased appetite, gradual lowering of weight, generalized weakness, and diffuse headache.

\section{Nursing diagnosis :}

I. Ineffective breathing pattern related to compression of lung secondary to liver metastases as manifested by increased respiratory rate.

II. Imbalanced nutrition less than body requirements related to anorexia secondary to liver metastases as manifested by weight loss

III. Fatigue related to poor intake secondary to anorexia as manifested by verbalization of patient.

\section{Nursing interventions:}

o Maintain fowlers position

○ Changed position $2^{\text {nd }}$ hourly.

o Encouraged to increase the food intake by advising the patient to take small frequent meals.

o Provided foods according to the preferences of the patient

o Administered Tab. Rantac 150 mg bd before meals.

- Monitored and recorded the fluid intake and output daily.

o Reduced noxious stimuli at the time of dining and water intake in between meals.

o Avoided strenuous exercises.

o Encouraged adequate periods of rest alternating with activity.

o Monitored for complications.

\section{Expected outcomes :}

○ The patient experiences improved respiratory status.

o Reports decreased shortness of breath.

o Exhibits normal respiratory rate with no adventitious sounds. 
$\circ$ Exhibits improved nutritional status by increased weight and improved laboratory data.

○ Reports improved appetite.

o Exhibits increased interest in activities and events.

o Plans activities to allow ample periods of rest.

\section{CONCLUSION :}

Most patients with carcinoid tumors seek treatment for metastatic disease. hence awareness should be created regarding carcinoid syndrome among the nursing professionals. The prognosis for patients with these tumors is variable and related to the site of the primary tumor. Comprehensive nursing care should be focused based on the site of metastases.

\section{Bibliography :}

Dan L. Longo (2010). Harrison's Hematology and Oncology; McGraw Hill Medical Publications;584-589.

J. Larry Jameson, Dennis L. Kasper et al. (2010). Harrison's Endocrinology. McGraw Hill Medical Publications; 354359

John A.A. Hunter, Nicholas A. Boon, et al. (2006). Davidson's Principles and Practice of Medicine. 20 ${ }^{\text {th }}$ edition; Churchill Livingstone Publications;791,903.

Suzanne c. Smeltzer, Brenda G. Bare et al. (2008). Brunner and Suddarth's Textbook of Medical and Surgical Nursing. $12^{\text {th }}$ edition; Lippincott Williams and Wilkins publications ;1323-1332. 